\title{
Imaging the Primary Cilium: Challenges and Approaches
}

\author{
Andrew Resnick
}

Department of Physics, Cleveland State University, Cleveland OH 44115

The primary cilium is an organelle hypothesized to be centrally important in many regulatory pathways driven by mechanosensation- typically involving sensing the apical fluid flow. Because the cilium is hypothesized to function based on its geometry- a slender cantilevered beam- obtaining knowledge of the dynamical shape is a key step in elucidating the physiological function. However, the cilium has a diameter well below the (optical) diffraction limit and grows parallel to the optical axis of a microscope. Both factors conspire to make imaging the living cilium a very challenging problem.

In my talk, I will discuss past and current methods used to increase the visibility of the primary cilium in live cell imaging. In addition, I will present reasons why obtaining data about the deformed shape of the cilium can be used to inform many disease models involving mechanosensation and/or muco-ciliary interactions. Finally, I will discuss my laboratory's use of optical trapping to both stimulate and measure the mechanical properties of the cilium. 